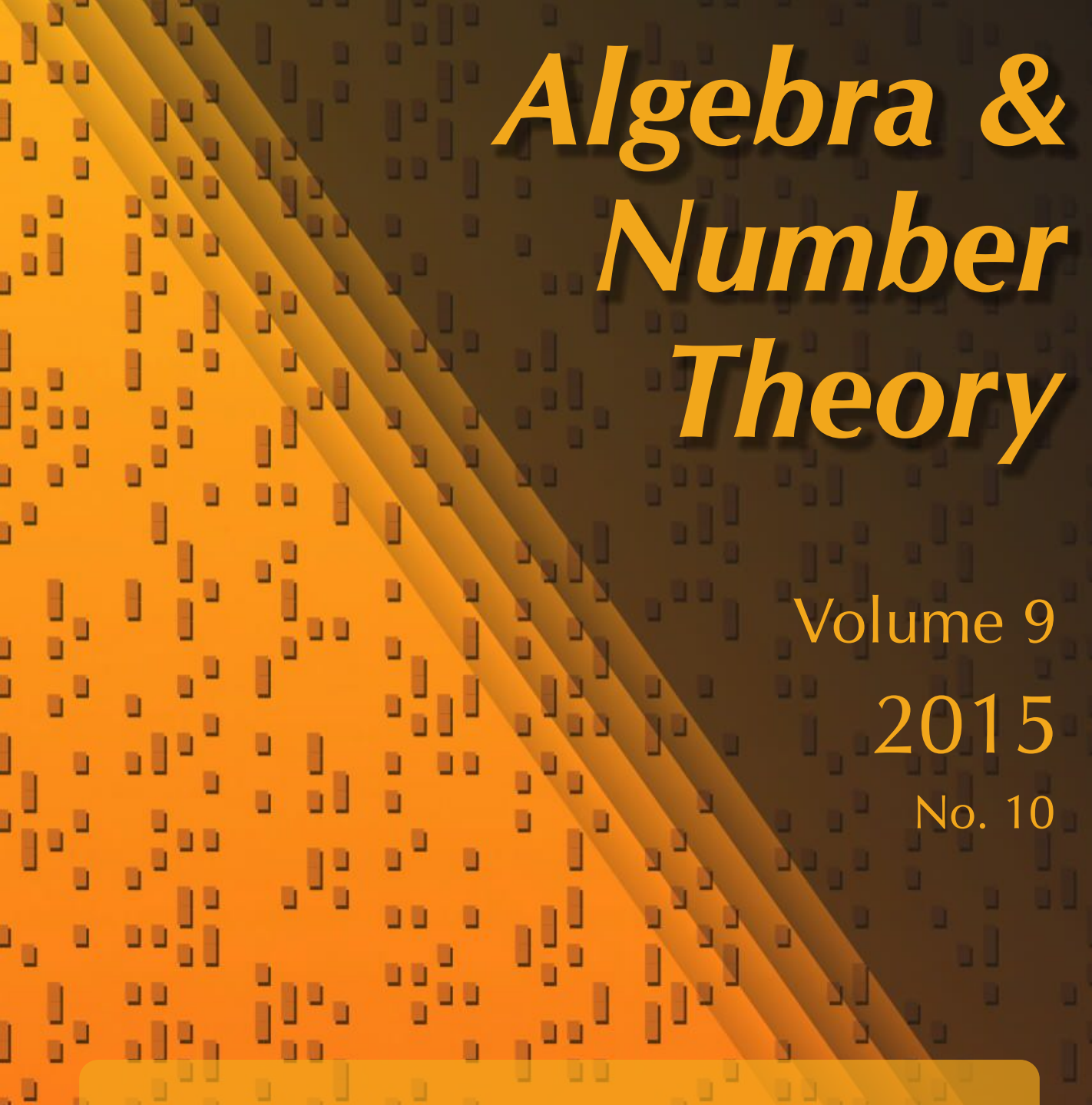

Polynomial values modulo primes on average and sharpness of the larger sieve

ـ. Xuancheng Shao

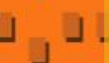

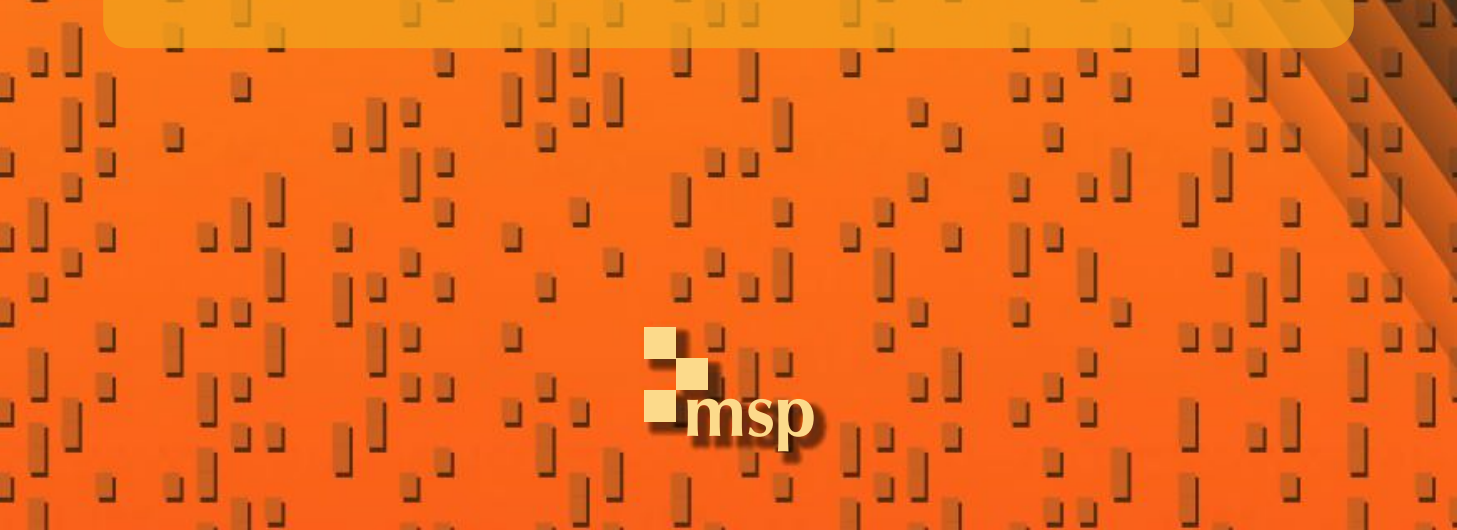




\title{
Polynomial values modulo primes on average and sharpness of the larger sieve
}

\author{
Xuancheng Shao
}

This paper is motivated by the following question in sieve theory. Given a subset $X \subset[N]$ and $\alpha \in\left(0, \frac{1}{2}\right)$. Suppose that $|X(\bmod p)| \leq(\alpha+o(1)) p$ for every prime $p$. How large can $X$ be? On the one hand, we have the bound $|X| \ll_{\alpha} N^{\alpha}$ from Gallagher's larger sieve. On the other hand, we prove, assuming the truth of an inverse sieve conjecture, that the bound above can be improved (for example, to $|X| \ll_{\alpha} N^{O\left(\alpha^{2014}\right)}$ for small $\alpha$ ). The result follows from studying the average size of $|X(\bmod p)|$ as $p$ varies, when $X=f(\mathbb{Z}) \cap[N]$ is the value set of a polynomial $f(x) \in \mathbb{Z}[x]$.

\section{Introduction}

For a positive integer $N$, denote by $[N]$ the set $\{1,2, \ldots, N\}$. The letter $p$ is always used to denote a prime. The primary goal of this paper is to study upper bounds for the sizes of subsets $X \subset[N]$ occupying a small fraction of residue classes modulo many primes $p$. Gallagher's larger sieve [1971] provides such an upper bound.

Theorem 1.1 (larger sieve). Let $X \subset[N]$ be a subset and $\mathscr{P}$ be a set of primes. We have

$$
|A| \leq \frac{\sum_{p \in \mathscr{P}} \log p}{\sum_{p \in \mathscr{P}}|X(\bmod p)|^{-1} \log p-\log N}
$$

whenever the denominator is positive.

See [Croot and Elsholtz 2004] for some variants of it and references therein for applications. We are particularly interested in the situation when $|X(\bmod p)| \leq \alpha p$ for some fixed $\alpha \in(0,1)$, and whether the bound provided by the larger sieve is best possible.

Corollary 1.2 (larger sieve, special case). Let $X \subset[N]$ be a subset and $\alpha \in\left(0, \frac{1}{2}\right]$. If $|X(\bmod p)| \leq(\alpha+o(1)) p$ for every prime $p$, then $|X| \ll N^{\alpha+o(1)}$.

MSC2010: primary 11N35; secondary 11R45, 11R09.

Keywords: Gallagher's larger sieve, inverse sieve conjecture, value sets of polynomials over finite fields. 
This is easily deduced from Theorem 1.1 by taking $\mathscr{P}$ to be the set of primes up to $N^{\alpha+o(1)}$. When $\alpha>\frac{1}{2}$, the statement still holds, but is beaten by the bound $|X| \ll_{\alpha} N^{1 / 2}$ following from the large sieve [Montgomery 1978]. When $\alpha \leq \frac{1}{2}$, is the bound $|X| \ll N^{\alpha+o(1)}$ sharp? If $X$ is the set of perfect squares up to $N$, then $|X| \sim N^{1 / 2}$ and $X$ occupies $(p+1) / 2$ residue classes (the quadratic residues) modulo any odd prime $p$. The question of whether this is the only type of sharp example is usually referred to as the inverse sieve conjecture, informally stated as follows.

Conjecture 1.3 (inverse sieve conjecture, rough form). Let $X \subset[N]$ be a subset. If $|X(\bmod p)| \leq 0.9 p$ for every prime $p$, then either one of the following two statements holds:

(1) The cardinality of $X$ is extremely small.

(2) The set $X$ possesses algebraic structure.

See Conjecture 4.1 below for one precise formulation of it. See also [Croot and Lev 2007; Helfgott and Venkatesh 2009; Walsh 2012; Green and Harper 2014] for more discussions and evidences towards it.

Now assume that $\alpha<\frac{1}{2}$ is fixed. Motivated by the inverse sieve conjecture, we consider the sizes of $X(\bmod p)$ when $X$ is the value set of a polynomial. For a polynomial $f(x) \in \mathbb{Z}[x]$ of degree $d \geq 1$, denote by $f_{p} \in \mathbb{F}_{p}[x]$ the reduction of $f$ modulo $p$. Let $\alpha_{p}(f)=p^{-1}\left|f_{p}\left(\mathbb{F}_{p}\right)\right|$, the relative size of the value set of $f(\bmod p)$. Define $\alpha(f)$ to be the average of $\alpha_{p}(f)$ as $p$ varies:

$$
\alpha(f)=\lim _{Q \rightarrow \infty} \frac{1}{\pi(Q)} \sum_{p \leq Q} \alpha_{p}(f) .
$$

Note the trivial lower bounds $\alpha_{p}(f) \geq d^{-1}$ and $\alpha(f) \geq d^{-1}$.

Theorem 1.4 (polynomial values modulo primes on average). Let $f \in \mathbb{Z}[x]$ be a polynomial of degree $d \geq 1$. Then

$$
\lim _{Q \rightarrow \infty} \frac{1}{\pi(Q)} \sum_{p \leq Q} \alpha_{p}(f)^{-1} \leq \tau(d),
$$

where $\tau(d)$ is the number of positive divisors of $d$. Consequently, $\alpha(f) \geq \tau(d)^{-1}$.

Note that for $d \geq 3$ we always have $\tau(d)<d$. Hence, it is reasonable to conjecture that Corollary 1.2 is not sharp whenever $\alpha$ is smaller than (and bounded away from) $\frac{1}{2}$. See the last section in [Shao 2014] for a preliminary discussion on the simplest case $d=3$.

Theorem 1.5 (inverse sieve conjecture implies improved larger sieve). Assume the truth of Conjecture 4.1. Let $X \subset[N]$ be a subset and $\alpha \in(0,1)$. Let $\epsilon \in(0,1)$ be a parameter. If $|X(\bmod p)| \leq(\alpha+o(1)) p$ for every prime $p$, then $|X| \ll_{\alpha, \epsilon} N^{1 / d}$, where $d$ is the smallest positive integer with $\tau(d) \geq(1-\epsilon) \alpha^{-1}$. 
For example, when $\alpha=0.49$, we obtain the upper bound $|X| \ll N^{1 / 4}$. On the other hand, since $\tau(d) \leq d^{C / \log \log d}$ for some constant $C>0$, we get $|X| \ll_{\alpha} N^{\alpha^{c \log \log \alpha^{-1}}}$ for some constant $c>0$, a huge improvement upon Corollary 1.2 for small $\alpha$ (assuming the truth of the inverse sieve conjecture).

Remark 1.6. Instead of assuming that $|X(\bmod p)| \leq(\alpha+o(1)) p$ for every prime $p$, knowing this on average over $p$ in an appropriate sense is sufficient for our proof to follow. In this paper, we will focus on the model case in which we assume the pointwise estimate.

In the remainder of this introduction we discuss further the quantities $\alpha_{p}(f)$ and $\alpha(f)$. Note that (1-1) becomes an equality when $f(x)=x^{d}$. Indeed, in this case we have $\alpha_{p}(f) \sim(p-1, d)^{-1}$, and thus the average of $\alpha_{p}(f)^{-1}$ is equal to

$$
\frac{1}{\phi(d)} \sum_{a \in(\mathbb{Z} / d \mathbb{Z})^{\times}}(a-1, d)=\tau(d) .
$$

Note, however, that in this case the average of $\alpha_{p}(f)$ is equal to

$$
\alpha(f)=\frac{1}{\phi(d)} \sum_{a \in(\mathbb{Z} / d \mathbb{Z})^{\times}}(a-1, d)^{-1},
$$

which can be evaluated to $\phi(d) / d$ when $d$ is squarefree (and is at least $(\phi(d) / d)^{2}$ for any $d)$. Since $\phi(d) / d \gg(\log \log d)^{-1}$, the following construction provides polynomials $f$ with smaller $\alpha(f)$.

Theorem 1.7 (polynomials with small value sets modulo primes). Define a sequence of polynomials $\left\{f_{n}\right\}$ by

$$
f_{1}(x)=x^{2}, \quad f_{n+1}(x)=\left(f_{n}(x)+1\right)^{2} .
$$

Then $\alpha_{p}\left(f_{n}\right)=a_{n}$ provided that $p>2 f_{n-1}(0)+2$ when $n>1$, where the sequence $\left\{a_{n}\right\}$ is defined by

$$
a_{1}=\frac{1}{2}, \quad a_{n+1}=a_{n}-\frac{1}{2} a_{n}^{2} .
$$

Moreover, we have $a_{n} \leq 2 n^{-1}$ for each $n$.

See Remark 2.5 below for the reasoning behind this construction of $f_{n}$. It is easy to see heuristically why one expects the relation $a_{n+1}=a_{n}-\frac{1}{2} a_{n}^{2}$. Indeed, if we model the value set of $f_{n}(\bmod p)$ as a random subset $S \subset \mathbb{F}_{p}$ with each element $s \in$ $\mathbb{F}_{p}$ chosen in $S$ with probability $a_{n}$ independently at random, then for every quadratic residue $r$, the probability that $r$ can be written as $(s+1)^{2}$ for some $s \in S$ is $2 a_{n}-a_{n}^{2}$. Hence the expected size of the set $(S+1)^{2}$ is $\left(2 a_{n}-a_{n}^{2}\right) p / 2+O(1)$, as desired.

Since $\operatorname{deg} f_{n}=2^{n}$, we have $\alpha\left(f_{n}\right) \ll\left(\log \left(\operatorname{deg} f_{n}\right)\right)^{-1}$. We do not know whether this is the best example or whether the bound for $\alpha(f)$ in Theorem 1.4 is sharp. See Section 6B for more discussion of this. 
The investigation of $\alpha_{p}(f)$ for a fixed prime $p$ has a long history (see [Birch and Swinnerton-Dyer 1959; Cohen 1970]), and explicit formulae for $\alpha_{p}(f)$ are known in terms of the proportion of fixed-point-free elements in a certain Galois group (see Lemma 5.1 and the remark following). Not surprisingly, the quantity $\alpha(f)$ can also be evaluated in terms of a certain Galois group, and this is recorded in Proposition 6.1. Due to a lack of understanding of the relevant Galois groups, our lower bound for $\alpha(f)$ is instead obtained by studying the number of solutions to $f(x) \equiv f(y)(\bmod p)$ on average as $p$ varies (see Section 2$)$, and it is for this reason that the average of $\alpha_{p}(f)^{-1}$ naturally shows up.

A related line of work is on classifying those polynomials $f \in \mathbb{F}_{p}[x]$ for which $\alpha_{p}(f)$ is close to the lower bound $d^{-1}$ (for a fixed $p$ ). In particular, results in [Gomez-Calderon and Madden 1988] imply that $\alpha_{p}(f) \geq 2 d^{-1}+o(1)$ whenever $p \not \equiv \pm 1(\bmod d)$.

The rest of this paper is organized as follows. In Section 2 we state a general and quantitative version of Theorem 1.4 for polynomials over arbitrary number fields, and outline the proof strategy, with the details given in Section 3. In Section 4 we state a precise form of the inverse sieve conjecture and deduce Theorem 1.5. In Section 5, Theorem 1.7 is proved by computing relevant Galois groups. Finally, in Section 6, we make some further remarks concerning the larger sieve as well as the quantity $\alpha(f)$.

\section{Statement of results and proof strategy}

Notation. For a number field $K$, we denote by $O_{K}$ its ring of integers and by $\Delta_{K}$ its (absolute) discriminant. For a prime ideal $\mathfrak{p}$ in $\mathfrak{O}_{K}$, we use $\kappa_{\mathfrak{p}}$ to denote the residue field $O_{K} / \mathfrak{p}$ and $N(\mathfrak{p})=\left|\kappa_{\mathfrak{p}}\right|$ to denote the norm of $\mathfrak{p}$. For a polynomial $f$ with coefficients in $\mathscr{O}_{K}$, we use $f_{\mathfrak{p}}$ to denote its reduction modulo $\mathfrak{p}$.

As indicated in the introduction, we are mainly interested in studying the sizes of value sets of $f_{\mathfrak{p}}$ for one-variable polynomials $f$.

Definition 2.1. Let $K$ be a number field, and let $f(x) \in \mathscr{O}_{K}[x]$ be a polynomial. For any prime ideal $\mathfrak{p}$ in $O_{K}$, define

$$
\alpha_{\mathfrak{p}}(f)=N(\mathfrak{p})^{-1}\left|f_{\mathfrak{p}}\left(\kappa_{\mathfrak{p}}\right)\right| .
$$

This will be studied via the related quantity that measures the number of solutions of $g_{\mathfrak{p}}=0$ for (multivariable) polynomials $g$.

Definition 2.2. Let $K$ be a number field and let $g(\underline{X}) \in \mathcal{O}_{K}[\underline{X}]$ be a polynomial in $n$ variables $\underline{X}=\left(X_{1}, \ldots, X_{n}\right)$. For any prime ideal $\mathfrak{p}$ in $\mathbb{O}_{K}$, define

$$
m_{\mathfrak{p}}(g)=N(\mathfrak{p})^{-(n-1)}\left|\left\{\underline{X} \in \kappa_{\mathfrak{p}}^{n}: g_{\mathfrak{p}}(\underline{X})=0\right\}\right| .
$$


To make our result quantitative, we also need a notion that measures the sizes of the coefficients of a polynomial.

Definition 2.3 (heights). Let $K$ be a number field, and let $g(x) \in \mathcal{O}_{K}[\underline{X}]$ be a polynomial. We define its (absolute logarithmic) height $h(g)$ to be the sum

$$
h(g)=\sum_{v} \max _{a} \log |a|_{v},
$$

where the sum is over all places $v$ of $K$ and the maximum is taken over all coefficients $a$ of $g$.

Here $|a|_{v}$ is the normalized absolute value, so that it does not depend on the choice of the field $K$. For example, when $f \in \mathbb{Z}[x]$ is primitive, the height $h(f)$ is the logarithm of the (usual archimedean) absolute value of the largest coefficient of $f$. See [Hindry and Silverman 2000] for basic properties of the height function.

Theorem 2.4. Let $K$ be a number field and $f \in \mathfrak{O}_{K}[x]$ be a polynomial of degree $d \geq 1$. Let $g \in \mathfrak{O}_{K}[x, y]$ be the polynomial defined by $g(x, y)=f(x)-f(y)$. Let $s(g)$ be the number of irreducible factors of $g$ in $K[x, y]$. Then for any $Q \geq 2$ we have

$$
\sum_{N(\mathfrak{p}) \leq Q} m_{\mathfrak{p}}(g)=s(g) \sum_{N(\mathfrak{p}) \leq Q} 1+O(Q \exp (-c \sqrt{\log Q})+h(g))
$$

for sufficiently small $c=c(K, d)>0$.

Proof of Theorem 1.4 assuming Theorem 2.4. First, by an application of the CauchySchwarz inequality, we have $\alpha_{\mathfrak{p}}(f) \geq m_{\mathfrak{p}}(g)^{-1}$. It thus suffices to show that

$$
s(g) \leq \tau(d)
$$

when $K=\mathbb{Q}$. This follows by considering the homogeneous part of degree $d$ of $g(x, y)=f(x)-f(y)$. Indeed, since this homogeneous part is $a\left(x^{d}-y^{d}\right)$ for some $a \neq 0$, it factors into $\tau(d)$ irreducible factors over $\mathbb{Q}$ (which are cyclotomic polynomials), and thus $g(x, y)$ can be factored into at most $\tau(d)$ irreducible factors over $\mathbb{Q}$.

Remark 2.5. The argument above motivates the choice of $f_{n}$ in Theorem 1.7. Indeed, if a polynomial $f$ is highly decomposable, in the sense that $f$ is the composition of many polynomials (each of which has degree at least 2 ), then $g(x, y)=f(x)-f(y)$ will necessarily have many irreducible factors, which should lead to small values of $\alpha_{p}(f)$. In Proposition 5.3 we deduce another consequence of Theorem 2.4, that indecomposable polynomials have large values of $\alpha_{p}$.

We will in fact prove the following more general result, of which Theorem 2.4 is a special case. Two polynomials $g_{1}, g_{2}$ are said to be equivalent if they are scalar multiples of each other. Recall also that $g \in K[\underline{X}]$ is said to be absolutely 
(or geometrically) irreducible if it is irreducible and remains irreducible over the algebraic closure of $K$.

Theorem 2.6 (average number of solutions modulo primes). Let $K$ be a number field and $g(\underline{X}) \in \mathbb{O}_{K}[\underline{X}]$ be a polynomial in $n$ variables of total degree $d \geq 1$. Let $s(g)$ be the number of nonequivalent irreducible factors of $g$ in $K[\underline{X}]$. Let $L$ be $a$ Galois extension of $K$ such that $g$ factors into absolutely irreducible factors in $L[\underline{X}]$. Let $C=C(K, n, d)>0$ be sufficiently large. If $Q \geq \exp \left(C\left(\log \Delta_{L}\right)^{2}\right)$, then

$$
\begin{aligned}
& \sum_{N(\mathfrak{p}) \leq Q} m_{\mathfrak{p}}(g) \log N(\mathfrak{p}) \\
& \quad=s(g) Q-t(g) \frac{Q^{\beta_{0}}}{\beta_{0}}+O\left(Q \exp (-c \sqrt{\log Q})+h(g)+\log \Delta_{L}\right)
\end{aligned}
$$

for sufficiently small $c=c(K, n, d)>0$, where $t(g) \in[0, s(g)]$, and the second term appears only if the Dedekind zeta function $\zeta_{L}$ has a Siegel zero $\beta_{0} \in\left(\frac{1}{2}, 1\right)$. Consequently, for $Q \geq \exp \left(C\left(\log \Delta_{L}\right)^{2}\right)$ we have

$$
\sum_{N(\mathfrak{p}) \leq Q} m_{\mathfrak{p}}(g) \leq s(g) \sum_{N(\mathfrak{p}) \leq Q} 1+O\left(Q \exp (-c \sqrt{\log Q})+h(g)+\log \Delta_{L}\right) .
$$

The bounds for the error terms stem from a quantitative version of the Chebotarev density theorem in [Lagarias and Odlyzko 1977]. Assuming the truth of the generalized Riemann hypothesis (GRH) for $\zeta_{L}$, we can get a much better error term $O\left(Q^{1 / 2}\left(\log \Delta_{L}+[L: Q] \log Q\right)\right)$, and of course without the Siegel zero term. The unconditional error term, however, is already enough for our application.

Proof of Theorem 2.4 assuming Theorem 2.6. We show that $g(x, y)=f(x)-f(y)$ factors into absolutely irreducible factors over $L=K\left(\mu_{d}\right)$, where $\mu_{d}$ is the group of $d$-th roots of unity. Indeed, since the homogeneous part of degree $d$ of $f(x)-f(y)$ is $a\left(x^{d}-y^{d}\right)$ for some nonzero $a \in K$, it factors over $L$ into linear factors. Thus, there is a factorization

$$
f(x)-f(y)=\prod_{i=1}^{r} g_{i}(x, y)
$$

of $f(x)-f(y)$ into absolutely irreducible factors $g_{1}, g_{2}, \ldots, g_{r}$, such that the top-degree part of each $g_{i}$ is defined over $L$. We claim that each $g_{i}$ is defined over $L$ as well. Suppose not. Without loss of generality, assume that some coefficient of $g_{1}$ does not lie in $L$. Let $\tau \in \operatorname{Gal}(\overline{\mathbb{Q}} / L)$ be an automorphism that moves this coefficient. Let $\tau\left(g_{1}\right)$ be the polynomial obtained by applying $\tau$ to every coefficient of $g_{1}$. Then $\tau\left(g_{1}\right)$ is also a factor of $f(x)-f(y)$, and thus $\tau\left(g_{1}\right)$ is equivalent to $g_{i}$ for some $1 \leq i \leq r$. By our choice of $\tau, \tau\left(g_{1}\right)$ must be equivalent to $g_{i}$ for some $i>1$, and thus $g_{1}$ and $g_{i}$ have equivalent top-degree parts. This contradicts the fact that $x^{d}-y^{d}$ has no repeated factors. 
Now that the potential Siegel zero $\beta_{0}$ of $\zeta_{L}$ depends only on $K$ and $d$, the Siegel zero term in (2-3) can be absorbed into the error term, and the conclusion follows easily from partial summation.

Remark 2.7. In the argument above we used the fact that polynomials of the form $f(x)-f(y) \in K[x, y]$ factor into absolutely irreducible factors in $L[x, y]$, with $L=K\left(\mu_{d}\right)$. For a general polynomial $g(\underline{X}) \in K[\underline{X}]$ of height $h(g)$, it can be shown that one can take $L$ with $[L: \mathbb{Q}] \leq C$ and $\Delta_{L} \leq C \exp (C h(g))$ for some constant $C=C(K, n, d)>0$. Thus the $\log \Delta_{L}$ factor in the error term can be removed, and the assumption on $Q$ can be replaced by $Q \geq \exp \left(C h(g)^{2}\right)$. We will, however, not need this relation between the size of $L$ and the height $h(g)$.

Remark 2.8. The arguments used in proving Theorem 2.6 can be generalized to study the average behavior of $\left|V\left(\mathbb{F}_{p}\right)\right|$ as $p$ varies, for any algebraic variety $V$ defined over $\mathbb{Z}$. More precisely, let $m=\operatorname{dim} V$. Then the average of $p^{-m}\left|V\left(\mathbb{F}_{p}\right)\right|$ as $p$ varies is equal to the number of irreducible components of $V$.

To finish this section, we sketch the proof of Theorem 2.6. By Lang-Weil, $m_{\mathfrak{p}}(g)$ is essentially the number of absolutely irreducible factors of $g_{\mathfrak{p}}$. Factor $g$ into absolutely irreducible factors in $L[\underline{X}]$, and consider the natural action of the Galois group $G=\operatorname{Gal}(L / K)$ on these factors. For almost all primes $\mathfrak{P} \subset O_{L}$, these absolutely irreducible factors remain absolutely irreducible modulo $\mathfrak{P}$, and thus $m_{\mathfrak{p}}(g)$ is essentially the number of these factors which are defined over $\kappa_{\mathfrak{p}}$. This is equal to the number of fixed points of the Frobenius element associated with $\mathfrak{P}$. By the Chebotarev density theorem, these Frobenius elements are equidistributed in $G$ as $\mathfrak{P}$ varies. Hence the average of $m_{\mathfrak{p}}(g)$ is equal to the average number of fixed points of the $G$-action. By Burnside's lemma, this is equal to the number of $G$-orbits, which is exactly the number of irreducible factors $s(g)$ of $g$. In carrying out this procedure some additional effort is needed to keep track of the explicit dependence on the height of $g$.

\section{Proof of Theorem 2.6}

In this section we prove Theorem 2.6. The implied constants appearing in this section are always allowed to depend on $K, n, d$.

Factor $(g)$ into principal prime ideals in $L[\underline{X}]$ :

$$
(g)=\left(g_{1}\right)^{e_{1}}\left(g_{2}\right)^{e_{2}} \cdots\left(g_{r}\right)^{e_{r}},
$$

where $g_{i} \in L[\underline{X}]$ is absolutely irreducible, and $g_{i}, g_{j}$ are not equivalent when $i \neq j$. Let $G$ be the Galois group $\operatorname{Gal}(L / K)$. For any $1 \leq i \leq r$ and any $\xi \in G$, let $\xi\left(g_{i}\right)$ be the polynomial obtained by applying $\xi$ to all coefficients of $g_{i}$. Since $\xi\left(g_{i}\right)$ is also a factor of $g, \xi\left(g_{i}\right)$ is equivalent to $g_{j}$ for some $1 \leq j \leq r$. Hence $\xi$ acts on 
$\left\{\left(g_{1}\right), \ldots,\left(g_{r}\right)\right\}$ by sending $\left(g_{i}\right)$ to $\left(\xi\left(g_{i}\right)\right)$. In this way we obtain a $G$-action on $\left\{\left(g_{1}\right), \ldots,\left(g_{r}\right)\right\}$.

Lemma 3.1 (Galois descent). Let $E$ be any field and $F$ be a Galois extension of $E$. Let $h \in F[\underline{X}]$ be a polynomial. The following two statements are equivalent:

(1) The ideal $(h) \subset F[\underline{X}]$ is fixed by every element of $G=\operatorname{Gal}(F / E)$.

(2) The ideal ( $h)$ is defined over $E$. In other words, there exists a scalar $\alpha \in F^{\times}$ such that $\alpha h \in E[\underline{X}]$.

Proof. This is a standard result in the theory of Galois descent. For completeness, we give a proof here. Clearly (2) implies (1). Now assume that (1) holds, so that for each $\xi \in G$, we have $\xi(h)=c_{\xi} h$ for some $c_{\xi} \in F^{\times}$. The scalars $\left\{c_{\xi}: \xi \in G\right\}$ form a 1-cocycle $G \rightarrow F^{\times}$, and thus by Hilbert's Theorem 90 we have $c_{\xi}=\alpha / \xi(\alpha)$ for some $\alpha \in F^{\times}$. Now that $\xi(h)=\alpha h / \xi(\alpha)$, we conclude that $\xi(\alpha h)=\alpha h$ for each $\xi \in G$. Thus $\alpha h \in E[\underline{X}]$, as desired.

Lemma 3.2. Let the notation be as above. The number of orbits of the G-action on $\left\{\left(g_{1}\right),\left(g_{2}\right), \ldots,\left(g_{r}\right)\right\}$ is equal to $s(g)$.

Proof. Let $\mathscr{H}=\left\{h_{1}, h_{2}, \ldots, h_{s}\right\}$ be the set of nonequivalent irreducible factors of $g$ (well defined up to scalars in $K$ ), where $s=s(g)$. We construct a bijection between the set of orbits and $\mathscr{H}$.

Let $O \subset\left\{\left(g_{1}\right),\left(g_{2}\right), \ldots,\left(g_{r}\right)\right\}$ be a $G$-orbit, and let $h$ be the product of those $g_{i}$ with $\left(g_{i}\right) \in \mathcal{O}$. We claim that $(h)$ is defined over $K$, and moreover $(h)$ is a prime ideal in $K[\underline{X}]$ (hence $(h)=\left(h_{j}\right)$ for some $1 \leq j \leq s$ ). In fact, since any $\xi \in G$ permutes the factors in $\mathbb{O}$, the ideal $(h)$ is fixed by $\xi$. By Lemma 3.1, the ideal $(h)$ is defined over $K$. Now let $h^{\prime} \in K[\underline{X}]$ be a factor of $h$ (with positive degree), and let $\mathbb{O}^{\prime} \subset \mathcal{O}$ be the set of those $\left(g_{i}\right) \in \mathcal{O}$ dividing $h^{\prime}$. For any $\left(g_{i}\right) \in \mathbb{O}^{\prime}$ and any $\xi \in G$, $\xi\left(g_{i}\right)$ is also a factor of $h^{\prime}$ and thus $\left(\xi\left(g_{i}\right)\right) \in \mathbb{O}^{\prime}$. This shows that $G$ preserves $O^{\prime}$, and thus $\mathbb{O}^{\prime}=0$ and $\left(h^{\prime}\right)=(h)$. Hence $(h)$ is a prime ideal.

Conversely, let $h_{j} \in \mathscr{H}$ be an irreducible factor of $g$, and let 0 be the set of those $\left(g_{i}\right)$ dividing $h_{j}$. We claim that $O$ is a $G$-orbit, and moreover the product of those ideals in $\mathcal{O}$ is equal to $\left(h_{j}\right)$. In fact, for any $\xi \in G$ and $\left(g_{i}\right) \in \mathbb{O}$, the polynomial $\xi\left(g_{i}\right)$

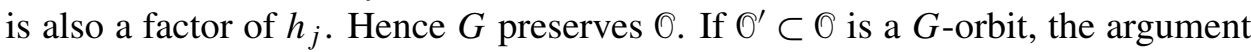
above shows that the product of the ideals in $O^{\prime}$ is defined over $K$. Hence $\mathbb{O}^{\prime}=0$ by the irreducibility of $h_{j}$. Finally, the argument above also shows that the product of the ideals in 0 is defined over $K$, and is thus equal to $\left(h_{j}\right)$.

The following lemma shows that the heights of the factors $g_{i}$ are controlled by the height of $g$. Note that the height $h\left(g_{i}\right)$ depends only on the ideal $\left(g_{i}\right)$ since two equivalent polynomials have the same height.

Lemma 3.3 (Gelfond's inequality). Let the notation be as above. Then $h\left(g_{i}\right) \leq$ $h(g)+C$ for some constant $C=C(K, n, d)>0$. 
Proof. See Proposition B.7.3 in [Hindry and Silverman 2000].

Let $\mathfrak{p}$ be a prime in $\mathscr{O}_{K}$ and $\mathfrak{P}$ be a prime in $\mathscr{O}_{L}$ lying above $\mathfrak{p}$. For each $1 \leq i \leq r$, let $\left(g_{i}\right)(\bmod \mathfrak{P})$ be the ideal in $\kappa_{\mathfrak{P}}[\underline{X}]$ obtained by reduction modulo $\mathfrak{P}$. The following lemma will be used to ensure that $\left(g_{i}\right)(\bmod \mathfrak{P})$ remains absolutely irreducible for all but finitely many $\mathfrak{P}$.

Lemma 3.4 (Noether). Let $n, d$ be positive integers. There exist polynomials $\ell_{1}, \ldots, \ell_{m}$ with integral coefficients depending only on $n$ and $d$ in variables $A_{i_{1} \ldots i_{n}}$ $\left(i_{1}+\cdots+i_{n} \leq d\right)$, such that the following statement holds. For any algebraically closed field $\bar{F}$, a polynomial $f \in \bar{F}[\underline{X}]$ in $n$ variables of total degree at most $d$ with

$$
f\left(x_{1}, \ldots, x_{n}\right)=\sum_{i_{1}+\cdots+i_{n} \leq d} a_{i_{1} \ldots i_{n}} x_{1}^{i_{1}} \cdots x_{n}^{i_{n}}
$$

is reducible over $\bar{F}$ or has total degree less than $d$ if and only if $\ell_{j}\left(\left(a_{i_{1} \ldots i_{n}}\right)\right)=0$ for each $1 \leq j \leq m$.

Proof. See Theorem 2A in [Schmidt 1976].

Lemma 3.5. Let the notation be as above. There exists a positive integer $E \leq$ $C \exp (C h(g))$, for some $C=C(K, n, d)>0$, such that $\left(g_{i}\right)(\bmod \mathfrak{P})$ is absolutely irreducible for each $1 \leq i \leq r$ whenever $\mathfrak{P} \nmid E$.

Proof. It suffices to prove the statement for each individual $i$. Let $\ell_{1}, \ldots, \ell_{m}$ be the polynomials in Lemma 3.4 corresponding to the degree of $g_{i}$. After normalizing we may assume that some coefficient of $g_{i}$ is equal to 1 . Thus $h(a) \leq h\left(g_{i}\right)$ for every coefficient $a$ of $g_{i}$, where $h(a)$ for $a \in L^{\times}$is defined by

$$
h(a)=\sum_{v} \max \left(\log |a|_{v}, 0\right) .
$$

Since $g_{i}$ is absolutely irreducible, $\ell_{j}$ does not vanish at the coefficient vector of $g_{i}$ for some $1 \leq j \leq m$; call this nonvanishing value $A \in L \backslash\{0\}$. Since all coefficients of $g_{i}$ have heights bounded by $h\left(g_{i}\right)$, we have $h(A)=O\left(h\left(g_{i}\right)+1\right)=O(h(g)+1)$. Therefore, there exists a positive integer $E \leq C \exp (C h(g))$ such that $A(\bmod \mathfrak{P})$ is nonzero whenever $\mathfrak{P} \nmid E$. For these $\mathfrak{P}$, the absolute irreducibility of $g_{i}(\bmod \mathfrak{P})$ follows from another application of Lemma 3.4.

Remark 3.6. The qualitative version of this statement is a special case of a general result in algebraic geometry: if $R$ is a domain with fraction field $F$ and $S$ is a domain finitely generated over $R$ such that the $F$-algebra $S_{F}=F \otimes_{R} S$ is absolutely irreducible over $F$, then there is a nonempty open subset $U \subset \operatorname{Spec}(R)$ such that the fiber algebra $S_{u}=k(u) \otimes_{R} S$ over $k(u)$, the residue field at $u$, is absolutely irreducible. 
Let $E$ be the positive integer from Lemma 3.5. After enlarging $E$ if necessary (but still with $E \leq C \exp (C h(g)))$, we may assume that $g_{1}(\bmod \mathfrak{P}), \ldots, g_{r}(\bmod \mathfrak{P})$ are pairwise inequivalent whenever $\mathfrak{P} \nmid E$.

Let $\mathfrak{p} \nmid E$ be a prime in $\mathscr{O}_{K}$ and $\mathfrak{P}$ be a prime in $O_{L}$ lying above $\mathfrak{p}$. The decomposition group $G_{\mathfrak{P}}=\operatorname{Gal}\left(\kappa_{\mathfrak{P}} / \kappa_{\mathfrak{p}}\right)$ acts on the factors $\left\{g_{1}(\bmod \mathfrak{P}), \ldots, g_{r}(\bmod \mathfrak{P})\right\}$ so that $\xi\left(g_{i}(\bmod \mathfrak{P})\right)$ is equivalent to $g_{j}(\bmod \mathfrak{P})$ for any $\xi \in G_{\mathfrak{P}}$. Comparing this with the $G$-action on $\left\{g_{1}, \ldots, g_{r}\right\}$ described at the beginning of this section, we see that they are compatible via the natural inclusion $G_{\mathfrak{P}} \hookrightarrow G$. In particular, both $G_{\mathfrak{P}}$ and $G$ can be viewed as subgroups of the symmetric group on $r$ elements.

For any conjugacy class $[\xi] \subset G$, let $s([\xi])$ be the number of fixed points of any element in $[\xi]$.

Lemma 3.7. Let the notation be as above. If $\mathfrak{p} \nmid E$ and $\mathfrak{p}$ is unramified in $L$, then $m_{\mathfrak{p}}(g)=s\left(\left[\sigma_{\mathfrak{p}}\right]\right)+O\left(N(\mathfrak{p})^{-1 / 2}\right)$, where $\left[\sigma_{\mathfrak{p}}\right]$ is the Frobenius conjugacy class associated to $\mathfrak{p}$.

Proof. Let $h \in\left\{g_{1}, \ldots, g_{r}\right\}$. Note that $\sigma_{\mathfrak{P}}$ fixes $(h)$ if and only if $\sigma_{\mathfrak{P}}$ fixes $\left(h_{\mathfrak{P}}\right)$, and this happens if and only if $\left(h_{\mathfrak{P}}\right)$ is defined over $\kappa_{\mathfrak{p}}$, by Lemma 3.1. Hence $s\left(\left[\sigma_{\mathfrak{p}}\right]\right)$ is exactly the number of nonequivalent absolutely irreducible factors of $g_{\mathfrak{p}}$ in $\kappa_{\mathfrak{p}}[\underline{X}]$.

Now let $h_{1}, \ldots, h_{s}, h_{s+1}, \ldots, h_{t}$ be the nonequivalent irreducible factors of $g_{\mathfrak{p}}$ in $\kappa_{\mathfrak{p}}[\underline{X}]$, where $h_{1}, \ldots, h_{s}$ are absolutely irreducible, with $s=s\left(\left[\sigma_{\mathfrak{p}}\right]\right)$. Let $V\left(h_{i}\right)$ be the solution set $\left\{\underline{X} \in \kappa_{\mathfrak{p}}^{n}: h_{i}(\underline{X})=0\right\}$. Then

$$
m_{\mathfrak{p}}(g)=N(\mathfrak{p})^{-(n-1)}\left(\sum_{i=1}^{t}\left|V\left(h_{i}\right)\right|+O\left(\sum_{i<j}\left|V\left(h_{i}\right) \cap V\left(h_{j}\right)\right|\right)\right) .
$$

For $i<j$, since $h_{i}$ and $h_{j}$ are nonequivalent, $V\left(h_{i}\right) \cap V\left(h_{j}\right)$ has dimension at most $n-2$. Thus by the Lang-Weil bound [1954] we have

$$
\left|V\left(h_{i}\right) \cap V\left(h_{j}\right)\right| \ll N(\mathfrak{p})^{n-2} .
$$

(In fact the weaker Schwarz-Zippel estimate is enough here). On the other hand, for $1 \leq i \leq s$, the Lang-Weil bound gives

$$
\left|V\left(h_{i}\right)\right|=N(\mathfrak{p})^{n-1}\left(1+O\left(N(\mathfrak{p})^{-1 / 2}\right)\right)
$$

since $h_{i}$ is absolutely irreducible, and for $s<i \leq t$ we have

$$
\left|V\left(h_{i}\right)\right|=\left|V\left(h_{i}\right) \cap V\left(h_{j}\right)\right| \ll N(\mathfrak{p})^{n-2}
$$

for some $s<j \leq t$, with $h_{j}$ a Galois conjugate of $h_{i}$. Combining these estimates together in (3-1) we obtain

$$
m_{\mathfrak{p}}(g)=s+O\left(N(\mathfrak{p})^{-1 / 2}\right),
$$

as desired. 
We are now ready to evaluate the quantity

$$
M_{f}(Q)=\sum_{N(\mathfrak{p}) \leq Q} m_{\mathfrak{p}}(g) \log N(\mathfrak{p})
$$

By Lemma 3.7, we have

$$
M_{f}(Q)=\sum_{\substack{N(\mathfrak{p}) \leq Q \\ \mathfrak{p} \text { unramified in } L}} s\left(\left[\sigma_{\mathfrak{p}}\right]\right) \log N(\mathfrak{p})+O\left(Q^{1 / 2} \log Q+\log E+\log \Delta_{L}\right)
$$

Since $E \leq C \exp (C h(g))$, we have $\log E=O(h(g)+1)$. Hence

$$
M_{f}(Q)=\sum_{C} s(C) \psi_{C}(Q)+O\left(Q^{1 / 2} \log Q+h(g)+\log \Delta_{L}\right),
$$

where the sum is over all conjugacy classes $C$ in $G$, and

$$
\psi_{C}(Q)=\sum_{\substack{N(\mathfrak{p}) \leq Q \\ \mathfrak{p} \text { unramified in } L \\\left[\sigma_{\mathfrak{p}}\right]^{m}=C}} \log N(\mathfrak{p}) .
$$

By (a quantitative version of) the Chebotarev density theorem [Lagarias and Odlyzko 1977], for $Q \geq \exp \left(C\left(\log \Delta_{L}\right)^{2}\right)$ we have

$$
\psi_{C}(Q)=\frac{|C|}{|G|} Q-\frac{|C|}{|G|} \chi_{0}(C) \frac{Q^{\beta_{0}}}{\beta_{0}}+O\left(Q \exp \left(-c(\log Q)^{1 / 2}\right)\right),
$$

where the second term occurs only if the Dedekind zeta function $\zeta_{L}$ has a Siegel zero $\beta_{0}$, and $\chi_{0}$ is the real character of a one-dimensional representation of $G$ for which the associated $L$-function has $\beta_{0}$ as a zero. It follows that

$$
\begin{aligned}
M_{f}(Q)=Q \cdot \frac{1}{|G|} \sum_{\xi \in G} s(\xi)-\frac{Q^{\beta_{0}}}{\beta_{0}} & \cdot \frac{1}{|G|} \sum_{\xi \in G} s(\xi) \chi_{0}(\xi) \\
& +O\left(Q \exp \left(-c(\log Q)^{1 / 2}\right)+h(g)+\log \Delta_{L}\right) .
\end{aligned}
$$

By Burnside's lemma and Lemma 3.2, we have

$$
\frac{1}{|G|} \sum_{\xi \in G} s(\xi)=s(g)
$$

The equality (2-3) follows by setting

$$
t(g)=\frac{1}{|G|} \sum_{\xi \in G} s(\xi) \chi_{0}(\xi) .
$$


By a change of summation, we can write

$$
t(g)=\frac{1}{|G|} \sum_{i=1}^{r} \sum_{\xi \in G_{i}} \chi_{0}(\xi),
$$

where $G_{i} \subset G$ is the subgroup of elements fixing $\left(g_{i}\right)$. Since $\chi_{0}$ is a one-dimensional real character, the inner sum is either 0 or $\left|G_{i}\right|$, and in the latter case $\chi_{0}(\xi)=1$ for all $\xi \in G_{i}$. Thus in the sum in (3-3) we may restrict to those $\xi$ with $\chi_{0}(\xi)=1$. Comparing this with (3-2), we obtain $t(g) \in[0, s(g)]$ as claimed.

Finally, the inequality (2-4) follows easily from (2-3) by dropping the Siegel zero term and partial summation.

\section{Inverse sieve conjecture implies improved larger sieve}

In this section we state a precise version of the inverse sieve conjecture and then prove Theorem 1.5. The implied constants here are always allowed to depend on $\alpha, \epsilon$.

Conjecture 4.1 (inverse sieve conjecture). Let $X \subset[N]$ be a subset and let $\epsilon, \epsilon^{\prime}>0$ be real. Assume that for each parameter $Q \geq N^{\epsilon}$ we have

$$
\sum_{p \leq Q} \frac{|X(\bmod p)|}{p} \leq\left(1-\epsilon^{\prime}\right) \pi(Q) .
$$

Then at least one of the following two situations happens:

(1) (very small size) $|X| \ll_{\epsilon, \epsilon^{\prime}} N^{\epsilon}$.

(2) (algebraic structure) There exists a polynomial $f(x) \in \mathbb{Q}[x]$ of degree $d \in$ $[2, C]$ and height at most $N^{C}$ such that $|X \cap f([N])| \geq C^{-1}|X|$, where $C=$ $C\left(\epsilon, \epsilon^{\prime}\right)$ is a constant.

Here, we say that a polynomial $f(x) \in \mathbb{Q}[x]$ has height at most $H$ if $f(x)=$ $A^{-1} f^{*}(x)$ for some positive integer $A \leq H$ and $f^{*} \in \mathbb{Z}[x]$ with all coefficients bounded by $H$ in absolute value. This is slightly different from the notion of height used in the statement of Theorem 2.4, in that $h(f)$ is invariant under scalar multiplication but the notion here is not. Note that if a polynomial $f(x) \in \mathbb{Q}[x]$ has height at most $H$, then $h(f) \ll \log H$.

Remark 4.2. We make a few remarks explaining why some quantitative aspects of this conjecture are reasonable.

- The condition on $X$ essentially says that $X$ misses a positive proportion of residue classes modulo primes $p$ on average, as soon as $p$ exceeds a small positive power of $N$. With this assumption, we know from the large sieve that $|X| \ll N^{1 / 2}$ and from the larger sieve that $|X| \ll N^{\alpha+O(\epsilon)}$ if the upper bound $\left(1-\epsilon^{\prime}\right) \pi(Q)$ is 
replaced by $\alpha \pi(Q)$. Without the knowledge about $X(\bmod p)$ for $p \leq N^{\epsilon}$, one can essentially add to $X$ any $N^{\epsilon}$ extra elements without violating the assumption, but one should still expect to see algebraic structure apart from these extra elements.

- The conclusion $|X \cap f([N])| \geq C^{-1}|X|$ is equivalent to the seemingly weaker one $|X \cap f(\mathbb{Q})| \geq C^{-1}|X|$, after a suitable modification of the polynomial $f$ which does not increase its height too much. To see that the interval $[N]$ can be replaced by $\mathbb{Z}$, note that the set $J=\{n \in \mathbb{Z}: 1 \leq f(n) \leq N\}$ is the union of at most $d$ intervals and has size at most $d N$. Since $X \cap f(\mathbb{Z})=X \cap f(J)$, there is an interval $I \subset J$ with $|X \cap f(I)| \geq d^{-1}|X \cap f(\mathbb{Z})|$, and we may assume that $I \subset[N]$ after a translation. To see that $f(\mathbb{Z})$ can be replaced by $f(\mathbb{Q})$, note that if $f(x) \in \mathbb{Z}$ for some $x \in \mathbb{Q}$ then the denominator of $x$ must divide some positive integer $B$ depending on the coefficient of $f$. Then $f(\mathbb{Q}) \cap \mathbb{Z} \subset f^{*}(\mathbb{Z}) \cap \mathbb{Z}$, where $f^{*}$ is defined by $f^{*}(x)=f(x / B)$.

- The conclusion that $f(\mathbb{Z})$ captures a positive proportion of $X$ cannot be replaced by the stronger one that $f(\mathbb{Z})$ captures almost all of $X$. Indeed, it is possible for $X$ to be the union of $f(\mathbb{Z})$ for several distinct polynomials $f$.

If $|X(\bmod p)| \leq \alpha p$ for small $\alpha$, repeated applications of Conjecture 4.1 allow us to strengthen it by requiring the degree $d$ to be fairly large.

Proposition 4.3 (inverse sieve conjecture in the larger sieve regime). Assume the truth of Conjecture 4.1. Let $X \subset[N]$ be a subset. Let $\alpha \in(0,1)$ and $\epsilon \in(0, \alpha)$ be real. Assume that $|X(\bmod p)| \leq(\alpha+o(1)) p$ for each prime $p$. Then at least one of the following two situations happens:

(1) (very small size) $|X| \ll_{\epsilon} N^{\epsilon}$.

(2) (algebraic structure) There exists a polynomial $f(x) \in \mathbb{Q}[x]$ of degree $d \in$ $[2, C]$ and height at most $N^{C}$ such that $|X \cap f(\mathbb{Z})| \geq C^{-1}|X|$, where $C=C(\epsilon)$ is a constant. Moreover, we may ensure that $\tau(d) \geq(1-\epsilon) \alpha^{-1}$.

Proof. Suppose that $|X| \gg N^{\epsilon}$. We will apply Conjecture 4.1 iteratively to construct a sequence of polynomials $f_{1}, f_{2}, \ldots, f_{k}$ and a sequence of sets $X_{0}=$ $X, X_{1}, X_{2}, \ldots, X_{k}$, with $k=O(1)$, such that the following conditions hold:

(1) $\operatorname{deg} f_{i}=d_{i} \in[2, C]$, and $\tau\left(d_{1} d_{2} \cdots d_{k}\right) \geq(1-\epsilon) \alpha^{-1}$;

(2) the height of $f_{i}$ is at most $N^{O(1)}$ for each $1 \leq i \leq k$;

(3) $X_{i} \subset[N]$ and $\left|X_{i}\right| \gg\left|X_{i-1}\right|$ for each $1 \leq i \leq k$;

(4) $f_{i}\left(X_{i}\right) \subset X_{i-1}$ for each $1 \leq i \leq k$.

Suppose first that these objects are constructed. Let $f=f_{1} \circ f_{2} \circ \cdots \circ f_{k}$. By property (1), the degree $d$ of $f$ is $O(1)$ and satisfies $\tau(d) \geq(1-\epsilon) \alpha^{-1}$. By 
property (2), the height of $f$ is $N^{O(1)}$. By property (3), we have $\left|X_{k}\right| \gg|X|$. By property (4), we have $f\left(X_{k}\right) \subset X \cap f([N])$. Hence

$$
|X \cap f([N])| \geq\left|f\left(X_{k}\right)\right| \gg\left|X_{k}\right| \gg|X|,
$$

as desired.

It thus remains to construct $f_{1}, \ldots, f_{k}$ and $X_{1}, \ldots, X_{k}$. Suppose that $f_{j}, X_{j}$ with $j<i$ are already chosen for some $i \geq 1$ satisfying the required properties (2)-(4) and $\operatorname{deg} f_{j}=d_{j} \in[2, C]$. We will construct $f_{i}$ and $X_{i}$ from these. Let $F=f_{1} \circ \cdots \circ f_{i-1}$ if $i>1$ and let $F$ be the identity map if $i=1$. Let $D$ be the degree of $F$. We may assume that $\tau(D)<(1-\epsilon) \alpha^{-1}$, since we may stop the iteration otherwise. By property (4), we have $F\left(X_{i-1}\right) \subset X$.

Let $F=A^{-1} F^{*}$ with $A \leq N^{C}$ a positive integer and $F^{*} \in \mathbb{Z}[x]$ a polynomial whose coefficients are all bounded by $N^{C}$. Let $G \in \mathbb{Z}[x, y]$ be the polynomial defined by $G(x, y)=F^{*}(x)-F^{*}(y)$. Let $p \nmid A$ be a prime. For each $r \in \mathbb{Z} / p \mathbb{Z}$, let $v_{p}(r)$ be the number of $x \in \mathbb{Z} / p \mathbb{Z}$ with $F(x) \equiv r(\bmod p)$. Then

$$
\begin{aligned}
\left|X_{i-1}(\bmod p)\right| & \leq \sum_{r \in F\left(X_{i-1}\right)(\bmod p)} v_{p}(r) \\
& \leq|X(\bmod p)|^{1 / 2}\left(\sum_{r} v_{p}(r)^{2}\right)^{1 / 2} \\
& \leq(\alpha+o(1))^{1 / 2} m_{p}(G)^{1 / 2} p,
\end{aligned}
$$

by Cauchy-Schwarz, the assumption that $|X(\bmod p)| \leq(\alpha+o(1)) p$, and the definition of $m_{p}(G)$ in Definition 2.2. For any $Q \geq N^{\epsilon}$, we then have

$$
\begin{aligned}
\sum_{p \leq Q} \frac{\left|X_{i-1}(\bmod p)\right|}{p} & \leq(\alpha+o(1))^{1 / 2} \sum_{p \leq Q} m_{p}(G)^{1 / 2}+O(\log A) \\
& \leq(\alpha+o(1))^{1 / 2} \pi(Q)^{1 / 2}\left(\sum_{p \leq Q} m_{p}(G)\right)^{1 / 2}+O(\log N) .
\end{aligned}
$$

Now apply Theorem 2.4 (and recall (2-2)) to obtain

$$
\begin{aligned}
& \sum_{p \leq Q} \frac{\left|X_{i-1}(\bmod p)\right|}{p} \\
& \quad \leq[(\alpha+o(1)) \tau(D)]^{1 / 2} \pi(Q)+O\left(Q \exp \left(-c(\log Q)^{1 / 2}\right)+Q^{1 / 2} \log N\right) .
\end{aligned}
$$

Since $\tau(D)<(1-\epsilon) \alpha^{-1}$, the first term above is at most $(1-\epsilon / 2) \pi(Q)$, and thus $X_{i-1}$ satisfies the hypotheses in Conjecture 4.1, with $\epsilon$ replaced by $\epsilon / 3$ and $N$ sufficiently large. Since $\left|X_{i-1}\right| \gg N^{\epsilon}$, we must be in the algebraic case. Let $f_{i} \in \mathbb{Q}[x]$ be a polynomial of degree $d_{i} \in[2, C]$ and height at most $N^{C}$ such that $\left|X_{i-1} \cap f_{i}([N])\right| \gg\left|X_{i-1}\right|$, and let $X_{i} \subset[N]$ be chosen so that $f_{i}\left(X_{i}\right) \subset X_{i-1}$ 
and $\left|X_{i}\right| \gg\left|X_{i-1}\right|$. This completes the inductive construction. Finally, since the quantity $\tau\left(d_{1} d_{2} \cdots d_{i}\right)$ strictly increases with $i$, the process terminates after $O(1)$ iterations.

Proof of Theorem 1.5. Apply Proposition 4.3 to conclude that either $|X|$ is very small and we are done, or else there exists a polynomial $f(x) \in \mathbb{Q}[x]$ of degree $d \in[2, C]$ and height at most $N^{C}$ such that $|X \cap f([N])| \geq C^{-1}|X|$. Moreover, we have $\tau(d) \geq(1-\epsilon) \alpha^{-1}$. Hence

$$
|X| \ll|X \cap f([N])| \leq|[N] \cap f([N])| \ll N^{1 / d},
$$

where the last inequality follows from a result of Walsh [2015], which removes the $\epsilon$ term from the exponent appearing in [Bombieri and Pila 1989; Heath-Brown 2002].

\section{Polynomials with small value sets modulo primes}

In this section we prove Theorem 1.7. First we state a result connecting the quantity $\alpha_{p}(f)$ to a Galois group. For a polynomial $f(x) \in \mathbb{F}_{p}[x]$ of degree $d$, denote by $R_{f}$ the set of roots in $\overline{\mathbb{F}_{p}(t)}$ of the polynomial $f(x)-t$. Define

$$
G_{f}=\operatorname{Gal}\left(\mathbb{F}_{p}\left(R_{f}\right) / \mathbb{F}_{p}(t)\right), \quad G_{f}^{*}=\operatorname{Gal}\left(\overline{\mathbb{F}}_{p}\left(R_{f}\right) / \overline{\mathbb{F}}_{p}(t)\right) .
$$

In other words, $G_{f}$ and $G_{f}^{*}$ are the Galois groups of the splitting field of $f(x)-t$ over $\mathbb{F}_{p}(t)$ and $\overline{\mathbb{F}}_{p}(t)$, respectively. It is easy to see that $G_{f}^{*}$ is a normal subgroup of $G_{f}$ with $G_{f} / G_{f}^{*}$ cyclic. In fact, $G_{f} / G_{f}^{*}$ is isomorphic to $\operatorname{Gal}\left(\mathbb{F}_{p}\left(R_{f}\right) \cap \overline{\mathbb{F}}_{p} / \mathbb{F}_{p}\right)$. For any subset $\Xi \subset G_{f}$, we use $\alpha(\Xi)$ to denote the proportion of elements in $\Xi$ with at least one fixed point under the natural action on $R_{f}$.

Lemma 5.1 [Cohen 1970]. Let $f(x) \in \mathbb{F}_{p}[x]$ be a polynomial of degree $d \geq 1$. Let $\sigma G_{f}^{*}$ be the coset which is the Frobenius generator of the cyclic quotient $G_{f} / G_{f}^{*}$, considered under its isomorphism with $\operatorname{Gal}\left(\mathbb{F}_{p}\left(R_{f}\right) \cap \overline{\mathbb{F}}_{p} / \mathbb{F}_{p}\right)$. Then

$$
\alpha_{p}(f)=\alpha\left(\sigma G_{f}^{*}\right)+O_{d}\left(p^{-1 / 2}\right) .
$$

In particular, if $G_{f}=G_{f}^{*}$ then

$$
\alpha_{p}(f)=\alpha\left(G_{f}\right)+O_{d}\left(p^{-1 / 2}\right) .
$$

Remark 5.2. In [Cohen 1970] this is deduced from a function field version of the Chebotarev density theorem. The Galois groups $G_{f}$ and $G_{f}^{*}$ above can be interpreted in terms of finite étale Galois coverings of $\mathbb{P}^{1}\left(\mathbb{F}_{p}\right)$. In this way Lemma 5.1 becomes a 0-dimensional special case of Deligne's equidistribution theorem. See [Kowalski 2010] for an excellent survey on this topic. This function field version of the Chebotarev density theorem and related equidistribution results play an important 
role in proving function field analogues of certain classical analytic number theory conjectures [Bank et al. 2015; Andrade et al. 2015; Entin 2014].

Proof of Theorem 1.7. Recall that the sequence of polynomials $\left\{f_{n}\right\}$ is defined by

$$
f_{1}(x)=x^{2}, \quad f_{n+1}(x)=\left(f_{n}(x)+1\right)^{2} .
$$

Write $G_{n}=G_{f_{n}}, G_{n}^{*}=G_{f_{n}}^{*}$, and $R_{n}=R_{f_{n}}$. Since any root $\alpha \in R_{n}$ satisfies either $f_{n-1}(\alpha)=-1+\sqrt{t}$ or $f_{n-1}(\alpha)=-1-\sqrt{t}$, we may decompose $R_{n}$ as the union $R_{n}=R_{n}^{+} \cup R_{n}^{-}$, with

$$
R_{n}^{ \pm}=\left\{\alpha \in R_{n}: f_{n-1}(\alpha)=-1 \pm \sqrt{t}\right\} .
$$

Note that both $\operatorname{Gal}\left(\mathbb{F}_{p}\left(R_{n}^{+}\right) / \mathbb{F}_{p}(\sqrt{t})\right.$ and $\operatorname{Gal}\left(\mathbb{F}_{p}\left(R_{n}^{-}\right) / \mathbb{F}_{p}(\sqrt{t})\right)$ are isomorphic to $G_{n-1}$, and similarly both $\operatorname{Gal}\left(\overline{\mathbb{F}}_{p}\left(R_{n}^{+}\right) / \overline{\mathbb{F}}_{p}(\sqrt{t})\right.$ and $\operatorname{Gal}\left(\overline{\mathbb{F}}_{p}\left(R_{n}^{-}\right) / \overline{\mathbb{F}}_{p}(\sqrt{t})\right)$ are isomorphic to $G_{n-1}^{*}$.

Let $H_{n}$ and $H_{n}^{*}$ be the normal subgroup of $G_{n}$ and $G_{n}^{*}$ that fixes $\sqrt{t}$, so that $\left[G_{n}: H_{n}\right]=\left[G_{n}^{*}: H_{n}^{*}\right]=2$. Since $H_{n}$ preserves both $R_{n}^{+}$and $R_{n}^{-}$, we get an embedding $\iota_{n}: H_{n} \hookrightarrow G_{n-1} \times G_{n-1}$ by setting the first and second components of $\iota_{n}(\xi)$ to be the images of $\xi$ under the two quotient maps $H_{n} \rightarrow \operatorname{Gal}\left(\mathbb{F}_{p}\left(R_{n}^{+}\right) / \mathbb{F}_{p}(\sqrt{t})\right)$ and $H_{n} \rightarrow \operatorname{Gal}\left(\mathbb{F}_{p}\left(R_{n}^{-}\right) / \mathbb{F}_{p}(\sqrt{t})\right)$, respectively. Similarly, we also get an embedding $\iota_{n}^{*}: H_{n}^{*} \hookrightarrow G_{n-1}^{*} \times G_{n-1}^{*}$.

We show, by induction on $n$, that when $p>2 f_{n-1}(0)+2$, the embeddings $\iota_{n}$ and $\iota_{n}^{*}$ are in fact isomorphisms, and moreover $G_{n}=G_{n}^{*}$ for each $n$. The base case is clear. Now assume that $G_{n-1}=G_{n-1}^{*}$. To see that $\iota_{n}^{*}$ is surjective, by Lemma 15 in [Fried 1970] it suffices to verify that for each $\lambda \in \overline{\mathbb{F}}_{p}$, at most one of the two values $-1+\sqrt{\lambda}$ and $-1-\sqrt{\lambda}$ is a branch point of $f_{n-1}$. By definition, the set of branch points of $f_{n-1}$ is

$$
\left\{f_{n-1}(x): x \in \overline{\mathbb{F}}_{p}, f_{n-1}^{\prime}(x)=0\right\} .
$$

This is easily computed to be the set

$$
\left\{f_{1}(0), f_{2}(0), \ldots, f_{n-1}(0)\right\}=\{0,1,4,25, \ldots\} .
$$

When $p>2 f_{n-1}(0)+2$, it is indeed the case that at most one of $-1+\sqrt{\lambda}$ and $-1-\sqrt{\lambda}$ can lie in this set for any $\lambda$. This shows that

$$
H_{n}^{*} \cong G_{n-1}^{*} \times G_{n-1}^{*} \cong G_{n-1} \times G_{n-1} .
$$

Moreover, since $H_{n}^{*} \subset H_{n} \subset G_{n-1} \times G_{n-1}$, we conclude that $H_{n}^{*}=H_{n}$, and thus $G_{n}^{*}=G_{n}$ as well. This completes the induction step.

With the structure of $G_{n}$ in hand, it is now a simple matter to write down the recursive relation

$$
\alpha_{p}\left(f_{n}\right)=\frac{1}{2}\left[1-\left(1-\alpha_{p}\left(f_{n-1}\right)\right)^{2}\right]=\alpha_{p}\left(f_{n-1}\right)-\frac{1}{2} \alpha_{p}\left(f_{n-1}\right)^{2},
$$


provided that $p>2 f_{n-1}(0)+2$. In fact, if $\xi \in G_{n}$ has a fixed point, then $\xi$ must fix $\sqrt{t}$ and thus lie in $H_{n}$, and moreover at least one of the two components of $\iota_{n}(\xi)$ has a fixed point. Finally, the bound $a_{n} \leq 2 n^{-1}$ follows from a standard induction argument.

We mentioned in Remark 2.5 the reasoning behind making $f_{n}$ highly decomposable. To end this section, we show that decomposability is quite essential in order for $\alpha_{p}$ to be small. The proof uses Theorem 2.4 together with results in [Fried 1970] (similar arguments are also used in [Guralnick and Wan 1997]). We say that a polynomial $f$ is indecomposable if it cannot be written as a composition of two polynomials of degree at least 2 .

Proposition 5.3. Let $f(x) \in \mathbb{Z}[x]$ be an indecomposable polynomial of degree $d \geq 1$. Then the average value of $\alpha_{p}(f)^{-1}$ as $p$ varies is at most 2 . Consequently, $\alpha(f) \geq \frac{1}{2}$.

Proof. Let $G$ be the Galois group of the splitting field of $f(x)-t$ over $\mathbb{Q}(t)$, viewed as a subgroup of the symmetric group $S_{d}$ on $d$ letters via its action on the $d$ roots of $f(x)-t$. Since $f$ is indecomposable, $G$ is primitive [Fried 1970, Lemma 2]. Moreover, $G$ contains a $d$-cycle [ibid., Lemma 3]. Hence either $d$ is prime or $G$ is doubly transitive [ibid., Lemma 7]. In either case, the conclusion follows from Theorem 2.4, since $\tau(d)=2$ when $d$ is prime and $(f(x)-f(y)) /(x-y) \in \mathbb{Q}[x, y]$ is irreducible when $G$ is doubly transitive [ibid., Lemma 14].

\section{Further remarks}

6A. More on the sharpness of Gallagher's larger sieve. Gallagher's larger sieve in its general form as stated in Theorem 1.1 has the optimal bound. Indeed, if we take $A \subset[N]$ to be any subset with cardinality $Q$ and take $\mathscr{P}$ be the set of all primes between $Q$ and $N$, the general form of the larger sieve gives the sharp bound $|A| \ll Q$, because the numerator is about $N$ and the denominator is about $N / Q$. This shows that any potential improvement to Corollary 1.2 must incorporate the ill-distribution modulo many small primes.

Under the assumption of Corollary 1.2, one may go over the argument in the proof of the larger sieve to find out what happens if $|A|$ is close to $N^{\alpha}$. Indeed, in the typical proof of Gallagher's larger sieve, one uses the upper and lower bounds

$$
\frac{|X|^{2}}{\alpha} \log Q \leq \sum_{x, x^{\prime} \in X} \sum_{\substack{p \mid x-x^{\prime} \\ p \leq Q}} \log p \leq|X|^{2} \log N+|X| Q,
$$

where $Q$ is about $N^{\alpha}$.

If the upper bound is (almost) sharp, then almost all of the nonzero differences $x-x^{\prime}$ should be $Q$-smooth, meaning that they do not have prime divisors larger 
than $Q$. For a random integer $n$, it is reasonable to expect that

$$
\sum_{\substack{p \mid n \\ p \leq Q}} \log p \approx \sum_{p \leq Q} \frac{\log p}{p} \sim \log Q .
$$

If this indeed holds for almost all differences $x-x^{\prime}$, then one can take $Q$ to be any small power of $N$ and deduce from (6-1) that $|X| \ll N^{\epsilon}$.

Now consider the situation when $X$ is the set of $d$-th powers up to $N$. Because of the factorization

$$
a^{d}-b^{d}=\prod_{\ell \mid d} \Phi_{\ell}(a, b),
$$

where $\Phi_{\ell}$ is the cyclotomic polynomial of degree $\phi(\ell)$, we cannot expect (6-2) to be true for $n=a^{d}-b^{d}$. However, it is still reasonable to expect that each factor $\Phi_{\ell}(a, b)$ satisfies (6-2). If so, then we obtain an upper bound in (6-1) with $\log N$ there replaced by $\tau(d) \log Q$, which in turn implies that $\tau(d) \geq \alpha^{-1}$. This is consistent with the conclusion of Theorem 1.5.

On the other hand, making this heuristic rigorous could be extremely hard. For example, it is an open problem to obtain a bound better than $|X| \ll N^{1 / 2}$ for $X \subset[N]$ with all nonzero differences $x-x^{\prime}\left(x, x^{\prime} \in X\right) N^{\kappa}$-smooth, where $\kappa>0$ is sufficiently small (see [Elsholtz and Harper 2015]).

There are versions of Gallagher's larger sieve over arbitrary number fields [Ellenberg et al. 2009; Zywina 2010]. One can ask similar questions about their sharpness in this general setting, and use Theorem 2.4 to formulate an improved larger sieve conjecture. We will not do so here since the case over $\mathbb{Z}$ is already quite interesting.

6B. Computing $\alpha(f)$ via Galois groups. The main result of this paper computes the average of $m_{p}(f)$ as $p$ varies, as a consequence of the Chebotarev density theorem. It is natural to ask if one can compute $\alpha(f)$, the average of $\alpha_{p}(f)$ as $p$ varies, directly, especially since we do have such a formula for each individual $\alpha_{p}(f)$ as in Lemma 5.1.

Proposition 6.1. Let $K$ be a number field and $f(x) \in \mathbb{O}_{K}[x]$ be a monic polynomial of degree d. Let $G=\mathrm{Gal}(K(R) / K(t))$, where $R$ is the set of roots of $f(x)-t$. Let $\alpha(G)$ be the proportion of elements in $G$ with at least one fixed point under the natural action on $R$. Then

$$
\lim _{Q \rightarrow \infty} \frac{1}{\pi(Q)} \sum_{N(\mathfrak{p}) \leq Q} \alpha_{\mathfrak{p}}(f)=\alpha(G) .
$$

In other words, $\alpha(f)=\alpha(G)$.

Remark 6.2. Unfortunately, we are unable to use this interpretation to obtain good lower bounds on $\alpha(f)$, but see [Guralnick and Wan 1997] for an example where 
large values of $\alpha_{p}(f)$ are studied via Galois groups. On the other hand, we feel that any possible improvement to the bound $\alpha(f) \geq \tau(d)^{-1}$ is likely to come from studying the Galois group $G$.

Proof. Write $E=K(R)$. Let $G^{*}=\operatorname{Gal}(\bar{K}(R) / \bar{K}(t))$. Let $L=E \cap \bar{K}$ be the algebraic closure of $K$ in $E$, so that $E=L(R)$ and $G^{*}=\operatorname{Gal}(E / L(t))$. By the primitive element theorem, there exists $\theta \in E$ such that $E=L(t, \theta)$. Suppose that $\theta$ satisfies the relation

$$
h_{m}(t) \theta^{m}+\cdots+h_{1}(t) \theta+h_{0}(t)=0,
$$

where $m=[E: L(t)]$ and $h_{m}(t), \ldots, h_{1}(t), h_{0}(t)$ are relatively prime polynomials over $L$. Let $h \in L[t, y]$ be the two-variable polynomial defined by

$$
h(t, y)=h_{m}(t) y^{m}+\cdots+h_{1}(t) y+h_{0}(t) .
$$

Clearly $h$ is a minimal polynomial of $\theta$, and thus $h$ is irreducible. By the definition of $L$, the polynomial $h$ is also absolutely irreducible.

Let $\mathfrak{p} \subset O_{K}$ be a prime in $K$ and $\mathfrak{P} \subset O_{L}$ be a prime in $L$ lying above $\mathfrak{p}$. By Lemma $3.5, h_{\mathfrak{P}} \in \kappa_{\mathfrak{P}}[t, y]$ remains absolutely irreducible for all but finitely many $\mathfrak{P}$. Let $\theta_{\mathfrak{P}} \in \overline{\kappa_{\mathfrak{P}}(t)}$ be an element satisfying $h_{\mathfrak{P}}\left(t, \theta_{\mathfrak{P}}\right)=0$, so that $E_{\mathfrak{P}}=\kappa_{\mathfrak{P}}\left(t, \theta_{\mathfrak{P}}\right)$ is a degree- $m$ field extension of $\kappa_{\mathfrak{P}}(t)$ with $E_{\mathfrak{P}} \cap \overline{\kappa_{\mathfrak{p}}}=\kappa_{\mathfrak{P}}$. Since $E / L(t)$ is Galois, all roots of $h(t, y)$ in $\overline{L(t)}$ lie in $E$. This implies that all roots of $h_{\mathfrak{P}}(t, y)$ in $\overline{\kappa_{\mathfrak{P}}(t)}$ lie in $E_{\mathfrak{P}}$ for all but finitely many $\mathfrak{P}$, and thus $E_{\mathfrak{P}} / \kappa_{\mathfrak{P}}(t)$ is also Galois. Note that there is a natural isomorphism $G^{*}=\operatorname{Gal}(E / L(t)) \cong \operatorname{Gal}\left(E_{\mathfrak{P}} / \kappa_{\mathfrak{P}}(t)\right)$, since an element in either Galois group is determined by its image of $\theta$ or $\theta_{\mathfrak{P}}$.

Now we look at the polynomial $f(x)-t$. Since it factors into linear factors over $E$, its reduction $f_{\mathfrak{p}}(x)-t$ factors into linear factors over $E_{\mathfrak{P}}$ for all but finitely many $\mathfrak{P}$. By an abuse of notation, we will continue to write $R$ for the set of roots of $f_{\mathfrak{p}}(x)-t$ in $\overline{\kappa_{\mathfrak{p}}(t)}$. Therefore the splitting fields $\kappa_{\mathfrak{P}}(R)$ and $\kappa_{\mathfrak{p}}(R)$ are contained in $E_{\mathfrak{P}}$. On the other hand, since $\theta \in K(R)$ and $L \subset K(R)$, we have $\theta_{\mathfrak{P}} \in \kappa_{\mathfrak{p}}(R)$ and $\kappa_{\mathfrak{P}} \subset \kappa_{\mathfrak{p}}(R)$ for all but finitely many $\mathfrak{P}$. This shows that $\kappa_{\mathfrak{p}}(R)=E_{\mathfrak{P}}$.

Let $\sigma_{\mathfrak{P}} G^{*}$ be the coset which is the inverse image of the Frobenius automorphism $\sigma_{\mathfrak{P}}$ under the quotient map

$$
\operatorname{Gal}\left(E_{\mathfrak{P}} / \kappa_{\mathfrak{p}}(t)\right) \rightarrow \operatorname{Gal}\left(\kappa_{\mathfrak{P}}(t) / \kappa_{\mathfrak{p}}(t)\right)=\operatorname{Gal}\left(\kappa_{\mathfrak{P}} / \kappa_{\mathfrak{p}}\right),
$$

which has kernel $\operatorname{Gal}\left(E_{\mathfrak{P}} / \kappa_{\mathfrak{P}}(t)\right)=G^{*}$. By Lemma 5.1, we have

$$
\alpha_{\mathfrak{p}}(f)=\alpha\left(\sigma_{\mathfrak{P}} G^{*}\right)+O_{d}\left(N(\mathfrak{p})^{-1 / 2}\right) .
$$

Note that the quantity $\alpha\left(\sigma_{\mathfrak{P}} G^{*}\right)$ does not depend on the choice of $\mathfrak{P}$. Via the inclusion $\operatorname{Gal}\left(\kappa_{\mathfrak{P}} / \kappa_{\mathfrak{p}}\right) \hookrightarrow \operatorname{Gal}(L / K)$, we may view $\sigma_{\mathfrak{P}}$ as an element in $\operatorname{Gal}(L / K)$ 
and $\sigma_{\mathfrak{P}} G^{*}$ as a coset in $G$. By the Chebotarev density theorem, the cosets $\sigma_{\mathfrak{P}} G^{*}$ become equidistributed in $G$ as $\mathfrak{p}$ varies. Therefore $\alpha(f)=\alpha(G)$ as desired.

For a generic polynomial of degree $d$, the Galois group $G$ in Proposition 6.1 is the full symmetric group $S_{d}$. Indeed, using the large sieve inequality, Gallagher [1973] obtained a precise bound on the number of exceptional polynomials (with coefficients bounded by a parameter) whose Galois group is not $S_{d}$. This bound has since been improved by Dietmann [2013]. By Proposition 6.1, this implies that

$$
\alpha(f)=\alpha\left(S_{d}\right)=1-\frac{1}{2}+\frac{1}{6}-\frac{1}{24}+\cdots+\frac{(-1)^{d-1}}{d !}
$$

for a typical $f$ of degree $d$. Moreover, this quantity tends to $1-e^{-1}$ as $d \rightarrow \infty$.

For $d \leq 4$, we have the following sharp lower bounds.

Proposition 6.3 (polynomials of small degree). For a positive integer $d$, let $\alpha_{d}$ be the smallest possible value of $\alpha(f)$, where $f \in \mathbb{Q}[x]$ is a polynomial of degree $d$. Then $\alpha_{2}=\frac{1}{2}, \alpha_{3}=\frac{2}{3}$, and $\alpha_{4}=\frac{3}{8}$.

Proof. For $d=2$ this is obvious. Suppose that $d \in\{3,4\}$. Let $G$ be the Galois group as in Proposition 6.1. We claim that $G \neq \mathbb{Z} / d \mathbb{Z}$, the cyclic group of order $d$. In fact, for $t \in \mathbb{Z}$ sufficiently large, the polynomial $f(x)=t$ has at least one real root and at least one nonreal root. Let $\alpha \in \mathbb{R}$ be a real root of $f(x)=t$. Then the splitting field of $f(x)-t$ contains properly the subfield $\mathbb{Q}(\alpha)$, and thus has degree larger than $d$ over $\mathbb{Q}$. This shows that the Galois group of $f(x)-t$ is not $\mathbb{Z} / d \mathbb{Z}$ for all $t$ sufficiently large. The fact that $G \neq \mathbb{Z} / d \mathbb{Z}$ then follows from Hilbert's irreducibility theorem. Now that $G \subset S_{d}$ is transitive and $G \neq \mathbb{Z} / d \mathbb{Z}$, the only possibilities are $G=S_{3}$ when $d=3$ and $G \in\left\{S_{4}, A_{4}, D_{4}\right\}$ when $d=4$. The conclusion follows by computing $\alpha(G)$ for these choices of $G$.

Not surprisingly, the nature of $\alpha_{d}$ depends not only on the size of $d$, but also the arithmetic of $d$ (see Proposition 5.3). In general, given a transitive subgroup $G \subset S_{d}$, we do not know how to tell whether $G$ can be realized as a Galois group as in Proposition 6.1. This is reminiscent of the inverse Galois problem over $K(t)$, but here we require the polynomial to take the shape $f(x)-t$ for some $f(x) \in K[x]$. We refer the interested reader to the book [Serre 2008] and references therein for background and known results on the classical inverse Galois problem.

\section{Acknowledgements}

Thanks to Brian Conrad for help with proofs and many useful comments, to Kannan Soundararajan for helpful discussions, to Akshay Venkatesh for asking a question that led to this paper, and to the anonymous referee for helpful suggestions. 


\section{References}

[Andrade et al. 2015] J. C. Andrade, L. Bary-Soroker, and Z. Rudnick, "Shifted convolution and the Titchmarsh divisor problem over $\mathbb{F}_{q}[t]$ ”, Philos. Trans. A 373:2040 (2015), 20140308, 18. MR 3338116

[Bank et al. 2015] E. Bank, L. Bary-Soroker, and L. Rosenzweig, "Prime polynomials in short intervals and in arithmetic progressions", Duke Math. J. 164:2 (2015), 277-295. MR 3306556 Zbl 06416949

[Birch and Swinnerton-Dyer 1959] B. J. Birch and H. P. F. Swinnerton-Dyer, "Note on a problem of Chowla", Acta Arith. 5 (1959), 417-423. MR 22 \#4675 Zbl 0091.04301

[Bombieri and Pila 1989] E. Bombieri and J. Pila, "The number of integral points on arcs and ovals", Duke Math. J. 59:2 (1989), 337-357. MR 90j:11099 Zbl 0718.11048

[Cohen 1970] S. D. Cohen, "The distribution of polynomials over finite fields", Acta Arith. 17 (1970), 255-271. MR 43 \#3234 Zbl 0209.36001

[Croot and Elsholtz 2004] E. S. Croot, III and C. Elsholtz, "On variants of the larger sieve", Acta Math. Hungar. 103:3 (2004), 243-254. MR 2005c:11113 Zbl 1060.11056

[Croot and Lev 2007] E. S. Croot, III and V. F. Lev, "Open problems in additive combinatorics", pp. 207-233 in Additive combinatorics, CRM Proc. Lecture Notes 43, Amer. Math. Soc., Providence, RI, 2007. MR 2009d:11038 Zbl 1183.11005

[Dietmann 2013] R. Dietmann, "Probabilistic Galois theory", Bull. Lond. Math. Soc. 45:3 (2013), 453-462. MR 3065016 Zbl 06176870

[Ellenberg et al. 2009] J. S. Ellenberg, C. Elsholtz, C. Hall, and E. Kowalski, "Non-simple abelian varieties in a family: geometric and analytic approaches", J. Lond. Math. Soc. (2) 80:1 (2009), 135-154. MR 2010f:11093 Zbl 1263.11064

[Elsholtz and Harper 2015] C. Elsholtz and A. J. Harper, "Additive decompositions of sets with restricted prime factors", Trans. Amer. Math. Soc. 367:10 (2015), 7403-7427. MR 3378834 Zbl 06479361

[Entin 2014] A. Entin, "On the Bateman-Horn conjecture for polynomials over large finite fields", preprint, 2014. arXiv 1409.0846

[Fried 1970] M. Fried, “On a conjecture of Schur”, Michigan Math. J. 17 (1970), 41-55. MR 41 \#1688 Zbl 0169.37702

[Gallagher 1971] P. X. Gallagher, "A larger sieve", Acta Arith. 18 (1971), 77-81. MR 45 \#214 Zbl 0231.10028

[Gallagher 1973] P. X. Gallagher, "The large sieve and probabilistic Galois theory", pp. 91-101 in Analytic number theory (St. Louis, MO, 1972), Proc. Sympos. Pure Math. 24, Amer. Math. Soc., Providence, R.I., 1973. MR 48 \#11020 Zbl 0279.10036

[Gomez-Calderon and Madden 1988] J. Gomez-Calderon and D. J. Madden, "Polynomials with small value set over finite fields", J. Number Theory 28:2 (1988), 167-188. MR 89d:11111 Zbl 0634.12014

[Green and Harper 2014] B. Green and A. J. Harper, "Inverse questions for the large sieve", Geom. Funct. Anal. 24:4 (2014), 1167-1203. MR 3248483 Zbl 1316.11085

[Guralnick and Wan 1997] R. Guralnick and D. Wan, "Bounds for fixed point free elements in a transitive group and applications to curves over finite fields", Israel J. Math. 101 (1997), 255-287. MR 98j:12002 Zbl 0910.11053

[Heath-Brown 2002] D. R. Heath-Brown, "The density of rational points on curves and surfaces", Ann. of Math. (2) 155:2 (2002), 553-595. MR 2003d:11091 Zbl 1039.11044 
[Helfgott and Venkatesh 2009] H. A. Helfgott and A. Venkatesh, "How small must ill-distributed sets be?”, pp. 224-234 in Analytic number theory, Cambridge Univ. Press, 2009. MR 2010d:11087 Zbl 1217.11073

[Hindry and Silverman 2000] M. Hindry and J. H. Silverman, Diophantine geometry, Graduate Texts in Mathematics 201, Springer, New York, 2000. MR 2001e:11058 Zbl 0948.11023

[Kowalski 2010] E. Kowalski, "Some aspects and applications of the Riemann hypothesis over finite fields", Milan J. Math. 78:1 (2010), 179-220. MR 2011g:11229 Zbl 1271.11113

[Lagarias and Odlyzko 1977] J. C. Lagarias and A. M. Odlyzko, "Effective versions of the Chebotarev density theorem", pp. 409-464 in Algebraic number fields: L-functions and Galois properties (Durham, UK, 1975), edited by A. Fröhlich, Academic Press, London, 1977. MR 56 \#5506 Zbl 0362.12011

[Lang and Weil 1954] S. Lang and A. Weil, "Number of points of varieties in finite fields", Amer. J. Math. 76 (1954), 819-827. MR 16,398d Zbl 0058.27202

[Montgomery 1978] H. L. Montgomery, "The analytic principle of the large sieve", Bull. Amer. Math. Soc. 84:4 (1978), 547-567. MR 57 \#5931 Zbl 0408.10033

[Schmidt 1976] W. M. Schmidt, Equations over finite fields: an elementary approach, Lecture Notes in Mathematics 536, Springer, Berlin, 1976. MR 55 \#2744 Zbl 0329.12001

[Serre 2008] J.-P. Serre, Topics in Galois theory, 2nd ed., Research Notes in Mathematics 1, A K Peters, Wellesley, MA, 2008. MR 2008i:12010 Zbl 1128.12001

[Shao 2014] X. Shao, "On an inverse ternary Goldbach problem", preprint, 2014. To appear in Amer. J. Math. arXiv 1404.6022

[Walsh 2012] M. N. Walsh, "The inverse sieve problem in high dimensions", Duke Math. J. 161:10 (2012), 2001-2022. MR 2954623 Zbl 06063227

[Walsh 2015] M. N. Walsh, "Bounded rational points on curves", Int. Math. Res. Not. 2015:14 (2015), 5644-5658. MR 3384452

[Zywina 2010] D. Zywina, "Hilbert's irreducibility theorem and the larger sieve", preprint, 2010. arXiv 1011.6465

Communicated by Andrew Granville

Received 2014-12-17 Revised 2015-07-19 Accepted 2015-08-17

xuancheng.shao@maths.ox.ac.uk Mathematical Institute, University of Oxford, Radcliffe Observatory Quarter, Woodstock Road, Oxford, OX2 6GG, United Kingdom 


\section{Algebra \& Number Theory}

msp.org/ant

\section{EDITORS}

MANAGING EDITOR

Bjorn Poonen

Massachusetts Institute of Technology

Cambridge, USA

\author{
EDITORIAL BOARD CHAIR \\ David Eisenbud \\ University of California \\ Berkeley, USA
}

BOARD OF EDITORS

Georgia Benkart

Dave Benson

Richard E. Borcherds

John H. Coates

J-L. Colliot-Thélène

Brian D. Conrad

Hélène Esnault

Hubert Flenner

Sergey Fomin

Edward Frenkel

Andrew Granville

Joseph Gubeladze

Roger Heath-Brown

Craig Huneke

Kiran S. Kedlaya

János Kollár

Yuri Manin

Philippe Michel
University of Wisconsin, Madison, USA

University of Aberdeen, Scotland

University of California, Berkeley, USA

University of Cambridge, UK

CNRS, Université Paris-Sud, France

Stanford University, USA

Freie Universität Berlin, Germany

Ruhr-Universität, Germany

University of Michigan, USA

University of California, Berkeley, USA

Université de Montréal, Canada

San Francisco State University, USA

Oxford University, UK

University of Virginia, USA

Univ. of California, San Diego, USA

Princeton University, USA

Northwestern University, USA

École Polytechnique Fédérale de Lausanne
Susan Montgomery

Shigefumi Mori

Raman Parimala

Jonathan Pila

Anand Pillay

Victor Reiner

Peter Sarnak

Joseph H. Silverman

Michael Singer

Vasudevan Srinivas

J. Toby Stafford

Ravi Vakil

Michel van den Bergh

Marie-France Vignéras

Kei-Ichi Watanabe

Efim Zelmanov

Shou-Wu Zhang
University of Southern California, USA

RIMS, Kyoto University, Japan

Emory University, USA

University of Oxford, UK

University of Notre Dame, USA

University of Minnesota, USA

Princeton University, USA

Brown University, USA

North Carolina State University, USA

Tata Inst. of Fund. Research, India

University of Michigan, USA

Stanford University, USA

Hasselt University, Belgium

Université Paris VII, France

Nihon University, Japan

University of California, San Diego, USA

Princeton University, USA

\section{PRODUCTION}

production@msp.org

Silvio Levy, Scientific Editor

See inside back cover or msp.org/ant for submission instructions.

The subscription price for 2015 is US $\$ 255 /$ year for the electronic version, and $\$ 440 /$ year ( $+\$ 55$, if shipping outside the US) for print and electronic. Subscriptions, requests for back issues and changes of subscribers address should be sent to MSP.

Algebra \& Number Theory (ISSN 1944-7833 electronic, 1937-0652 printed) at Mathematical Sciences Publishers, 798 Evans Hall \#3840, c/o University of California, Berkeley, CA 94720-3840 is published continuously online. Periodical rate postage paid at Berkeley, CA 94704, and additional mailing offices.

ANT peer review and production are managed by EditFLOW ${ }^{\circledR}$ from MSP.

\section{PUBLISHED BY}

- mathematical sciences publishers

nonprofit scientific publishing

http://msp.org/

(C) 2015 Mathematical Sciences Publishers 


\section{Algebra \& Number Theory}

Volume $9 \quad$ No. $10 \quad 2015$

Equivariant torsion and base change

MICHAEL LIPNOWSKI

Induction parabolique et $(\varphi, \Gamma)$-modules

CHRISTOPHE BREUIL

On the normalized arithmetic Hilbert function

MOUNIR HAJLI

SUNE PRECHT REEH

XUANCHENG SHAO

Bounds for Serre's open image theorem for elliptic curves over number fields

DAVIDE LOMBARDO

On 0-cycles with modulus

AMALENDU KRISHNA 